\title{
Efecto de las características de los polvos cerámicos sobre la densificación de materiales cerámicos PZT
}

\author{
L. A. CEli, A. C. CABAllero, M. VilleGAS, P. DURÁN, C. MOURE, J. F. FERNÁNDEZ. \\ Departamento de Electrocerámica, Instituto de Cerámica y Vidrio, CSIC. 28500 Arganda del Rey, Madrid.
}

\begin{abstract}
Con el fin de obtener un mayor control de las propiedades del titanato circonato de plomo (PZT) se ha usado el método de calcinación reactiva a partir de titanato de circonio y $\mathrm{PbO}$. En base a este procedimiento se han preparado polvos cerámicos de PZT, con composición en la frontera morfotrópica de fases, siguiendo tres procesos diferenciados de calcinación. Los polvos cerámicos obtenidos muestran una distribución de porosidad diferente y un tamaño de partícula promedio de $\sim 0.3 \mu \mathrm{m}$. Se ha encontrado que la distribución de porosidad del polvo cerámico tiene influencia en la densificación del material. La caída de densidad del PZT a alta temperatura está directamente relacionada con la volatilización del PbO durante la sinterización y con el área de poro de los polvos de partida.
\end{abstract}

Palabras clave: titanato circonato de plomo (PZT), porosidad, sinterización, cerámicas piezoeléctricas.

Effect of powder characteristics on densification of PZT ceramics.

Lead zirconate titanate (PZT) ceramic powders were processed following the reactive calcination method from zirconium titanate and $\mathrm{PbO}$. PZT ceramic powders with the morphotropic phase boundary composition were prepared by three different calcination processes. The so obtained ceramic powders have different porosity distribution with average particle size of $\sim 0.3$ $\mu \mathrm{m}$. It was found that the porosity distribution related to the synthesis process affects the densification of the ceramic. The density loss of PZT at high temperatures is linearly related to the $\mathrm{PbO}$ losses and the pore area of the starting powders.

Key words: lead zirconate titanate (PZT), porosity, sintering, piezoelectric ceramics.

\section{INTRODUCCION}

Los materiales cerámicos basados en titanato circonato de plomo, PZT, han sido utilizados como elementos piezoeléctricos en una amplia variedad de aplicaciones (1-5). En el rango de composición de la frontera morfotrópica de fases, estos materiales presentan coeficientes piezoeléctricos muy elevados (1). Los parámetros de procesamiento juegan un papel fundamental en la obtención de materiales densos con microestructura controlada, lo que permite la reproducibilidad y optimización de las propiedades de los materiales cerámicos PZT.

Cuando se realiza la síntesis de PZT mediante el proceso convencional de reacción en estado sólido a partir de la mezcla de los diferentes óxidos o carbonatos de partida, se obtienen polvos cerámicos poco reactivos. De esta forma es necesario el empleo de temperaturas elevadas para los procesos de sinterización del material que dan lugar a la formación de fases intermedias deletéreas para las propiedades (6-8). Estas dificultades conducen a la pérdida de la estequiometría por volatilización de $\mathrm{PbO}$, lo cual puede ocasionar la descomposición del material PZT (8-9). Como consecuencia se generan inhomogeneidades en la microestructura, que se traducen en una disminución de la densificación del material y en el deterioro de sus propiedades dieléctricas y piezoeléctricas.

El proceso de calcinación reactiva o de calcinación en dos etapas (10-13) permite la obtención de polvos cerámicos submicrónicos altamente reactivos que disminuyen las temperaturas de los procesos de sinterización. De esta forma se obtie- ne un mejor control de las pérdidas de $\mathrm{PbO}$ por volatilización y, por lo tanto, la inhibición de los fenómenos de descomposición de PZT. Este control estequiométrico favorece el desarrollo de una microestructura más homogénea que redunda en la mejora de las propiedades dieléctricas y piezoeléctricas del material.

Se han preparado polvos cerámicos de PZT con composición cercana a la frontera morfotrópica de fases por el método de calcinación reactiva, resultando tres distribuciones de porosidad diferentes. Las relaciones entre la distribución de porosidad del polvo cerámico obtenido con la microestructura y las propiedades dieléctricas y piezoeléctricas del material final son el objeto del presente trabajo.

\section{PROCEDIMIENTO EXPERIMENTAL}

Se han preparado polvos cerámicos de composición $\left[\mathrm{Pb}_{0.97} \mathrm{Sr}_{0.03}\left(\mathrm{Zr}_{0.5225} \mathrm{Ti}_{0.4625} \mathrm{Nb}_{0.015}\right)\right] \mathrm{O}_{3}$, PZT, mediante el método de calcinación reactiva en dos etapas (10). En la primera etapa se sintetizó el polvo cerámico $\left(\mathrm{Zr}_{0.5225} \mathrm{Ti}_{0.4625} \mathrm{Nb}_{0.015}\right) \mathrm{O}_{2}, \mathrm{ZT}$. Para ello se partió de la mezcla mecánica de los óxidos de $\mathrm{ZrO}_{2}$ (Sepr Saint-Gobain CS02), $\mathrm{TiO}_{2}$ (Thann et Mulhouse) y $\mathrm{Nb}_{2} \mathrm{O}_{5}$ (Fluka), obtenida por molienda durante 3 horas, en molino de bolas de $\mathrm{ZrO}_{2}$, en medio isopropílico, como en todas las moliendas realizadas a lo largo de este trabajo. Las cantidades de reactivos pesadas corresponden a las relaciones que 
se dan en la composición nominal anteriormente indicada. La mezcla peletizada se calcinó a $1400^{\circ} \mathrm{C}$ durante 4 horas y posteriormente fue molida en molino de bolas, y en molino de atricción, durante tres horas. En la segunda etapa de calcinación se siguieron tres procesos diferentes para la obtención de tres tipos de polvos cerámicos de PZT, a partir de la mezcla mecánica de $\mathrm{ZT}, \mathrm{PbO}$ (Aldrich) y $\mathrm{SrCO}_{3}$ (Carlo Erba), en las cantidades correspondientes a la composición nominal mencionada. El primer proceso consistió en una calcinación doble, primero a $725^{\circ} \mathrm{C} / 4$ horas y, tras una molienda en molino de bolas de $\mathrm{ZrO}_{2}$ durante 3 horas, se realizó una segunda calcinación a $750^{\circ} \mathrm{C} / 4$ horas (PZT-PIM). El segundo (PZT-PIB) y tercer proceso (PZT-PIA) se realizaron con una única etapa de calcinación a $775^{\circ} \mathrm{C} / 4$ horas, empleando una relación superficie/volumen del crisol cuatro veces mayor en el tercer proceso que en el segundo. Los polvos sintetizados fueron molidos en molino de bolas, $2 \mathrm{~h}$, y molino de atricción, $3 \mathrm{~h}$. Al final de cada etapa de calcinación se realizó la caracterización de los polvos cerámicos mediante las técnicas de Difracción de Rayos X (DRX), porosimetría de mercurio, BET (superficie específica), Microscopía Eléctronica de Barrido (MEB) y LáserCoulter (tamaño de partícula) . La técnica DRX se utilizó a fin de confirmar la completa reacción de PZT.

A fin de desaglomerar y disminuir el volumen de porosidad del polvo cerámico PZT-PIB, se turbinó a la velocidad de $10.000 \mathrm{rpm}$ durante 10 minutos en medio isopropílico. Los diferentes polvos cerámicos se prensaron uniaxialmente, a una presión de $45 \mathrm{MPa}$, en discos de $15 \mathrm{~mm}$ de diámetro y $1.5 \mathrm{~mm}$ de espesor. Los discos prensados se sinterizaron en un crisol sellado con $\mathrm{ZrO}_{2}$, y con una atmósfera de $\mathrm{PbO}$ creada por un tampón consistente en una mezcla $\mathrm{PbZrO}_{3}+5 \% \mathrm{ZrO}_{2}$. La sinterización de los discos se realizó en un rango de temperaturas de $1025^{\circ} \mathrm{C}$ a $1250^{\circ} \mathrm{C}$ y durante 2 horas. Sobre las muestras sinterizadas se determinó la densidad aparente mediante el método de Arquímedes. Las pérdidas de peso se determinaron por diferencias de peso entre el material en verde y el material sinterizado. Las microestructuras se observaron por MEB sobre la superficie de las muestras sinterizadas. El tamaño de grano se determinó por medio de un analizador de imágenes de

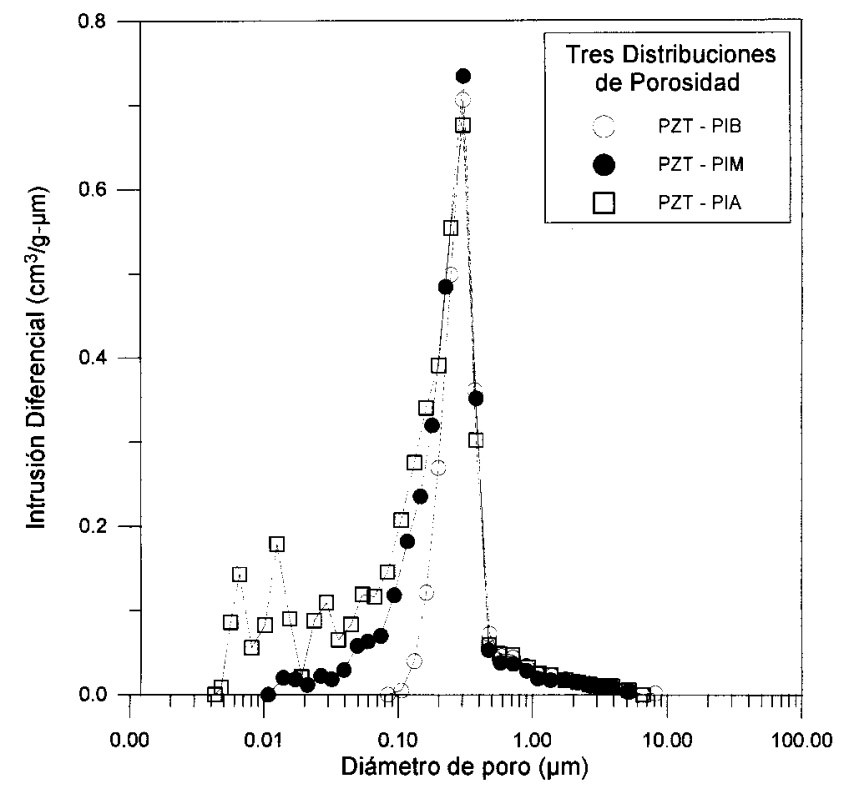

Figura 1. Distribuciones de tamaño de poro correspondientes a los tres polvos cerámicos PZT obtenidos.
TABla I. CARACTERísticas de los POlVOS CERÁMICOS PZT. d50 REPRESENTA EL TAMAÑO DE PARTíCULA PARA EL 50\% DE LA DISTRIBUCIÓN.

\begin{tabular}{|c|c|c|c|}
\hline Polvo Cerámico & $\begin{array}{c}\text { Superficie } \\
\text { Especifica } \\
\left(\mathbf{m}^{2} / \mathbf{g}\right)\end{array}$ & $\begin{array}{c}\text { Area Total } \\
\mathbf{d e} \text { Poros } \\
\left(\mathbf{m}^{2} / \mathbf{g}\right)\end{array}$ & $\begin{array}{c}\text { Tamaño de } \\
\text { Partícula } \mathbf{d}_{50} \\
(\boldsymbol{\mu} \mathbf{m})\end{array}$ \\
\hline PZT-PIB & $1.7 \pm 0.05$ & $2.0 \pm 0.05$ & $0.37 \pm 0.01$ \\
\hline PZT-PIM & $1.9 \pm 0.05$ & $2.6 \pm 0.05$ & $0.31 \pm 0.01$ \\
\hline PZT-PIA & $2.3 \pm 0.05$ & $3.8 \pm 0.05$ & $0.32 \pm 0.01$ \\
\hline
\end{tabular}

Princeton Gamma-Tech, Inc. (programa IMAGIST II). Este programa determina el tamaño promedio del diámetro de la superficie proyectada en un plano, por lo cual se le hizo la corrección del factor de forma de 1.5 para partícula esféricas. Para las medidas de propiedades dieléctricas se emplearon discos desvastados, con el fin de que sus caras sean planoparalelas, y electrodadas con pintura de plata. El electrodo fue sinterizado a $700^{\circ} \mathrm{C}$ durante 30 minutos. El proceso de polarización se realizó bajo un campo de $40 \mathrm{kV} / \mathrm{cm}$, a una temperatura de $120^{\circ} \mathrm{C}$ durante 30 minutos. Las propiedades piezoeléctricas se evaluaron de acuerdo con las normas IEEE (14), utilizando un analizador vectorial de impedancias HP 4192 A, controlado por un ordenador HP 9216. Para la determinación del coeficiente $\mathrm{d}_{33}$ se usó un equipo Berlincourt Piezo-d meter.

\section{RESULTADOS Y DISCUSIÓN}

Mediante los procesos de calcinación reactiva empleados se obtuvieron tres polvos con un tamaño de partícula similar (Tabla I) y una distribución de porosidad diferente (Figura 1). En las curvas de distribución de porosidad de los tres polvos cerámicos se pueden observar tres zonas. La primera zona corresponde a poros de gran tamaño (macroporos) entre 0.5 y 9 micras. Por comparación de estos macroporos con el tamaño de partícula promedio (Tabla I) se puede concluir que son de tipo interaglomerado. Los tres tipos de polvo cerámico presentan una distribución de poros similar en esta zona.

La segunda zona de distribución de poros (mesoporos) está comprendida entre $\sim 70 \mathrm{~nm}$ y $0.5 \mu \mathrm{m}$. Esta zona presenta una máxima velocidad de intrusión de mercurio que es análoga para los tres polvos en estudio y se corresponde con un tamaño de poro cercano a $0.3 \mu \mathrm{m}$, que es del mismo orden que el tamaño promedio de las partículas de los polvos cerámicos. Este dato indica que se trata de poros existentes entre las partículas de los polvos en consideración. El polvo cerámico PZT-PIB presenta la distribución de poros más estrecha; por el contrario, los otros dos polvos cerámicos presentan un ensanchamiento de esta zona debido a la presencia de una mayor cantidad de poros de menor tamaño, entre $70 \mathrm{~nm}$ y $100 \mathrm{~nm}$.

La tercera zona de distribución de poros (microporos), de tamaños entre $4 \mathrm{~nm}$ y $70 \mathrm{~nm}$, es la que fundamentalmente distingue a estas tres distribuciones. Puede observarse que el polvo PZT-PIB no presenta poros en esta zona, mientras que el polvo PZT-PIA exhibe una gran cantidad de estos microporos. La presencia de este tipo de poros podría atribuirse a dos posibles situaciones: a) grietas que se forman en el interior de las partículas como consecuencia de la gran expansión volumétrica (10) 
que se produce durante el proceso de síntesis del polvo cerámico, al incorporarse el ión $\mathrm{Pb}^{+2}$ en la celda cristalina de titanato de circonio previamente sintetizado y expandiéndola fuertemente. Las partículas resultantes se rompen fácilmente mediante el proceso de molienda altamente energético utilizado $(10,15,16)$. b) Deficiencia en la disposición o empaquetamiento de las partículas constituyentes del polvo cerámico, produciéndose microporos de tipo capilar debido a un deficiente contacto entre ellas. Los tres polvos cerámicos en estudio se diferencian fundamentalmente por el volumen de microporos. Las diferencias entre PZT-PIB y PZT-PIA se pueden atribuir a la relación superficie/volumen del crisol empleada, lo que provoca una atmósfera de calcinación distinta. Para PZT-PIM las diferencias se atribuyen al doble proceso de calcinación empleado.
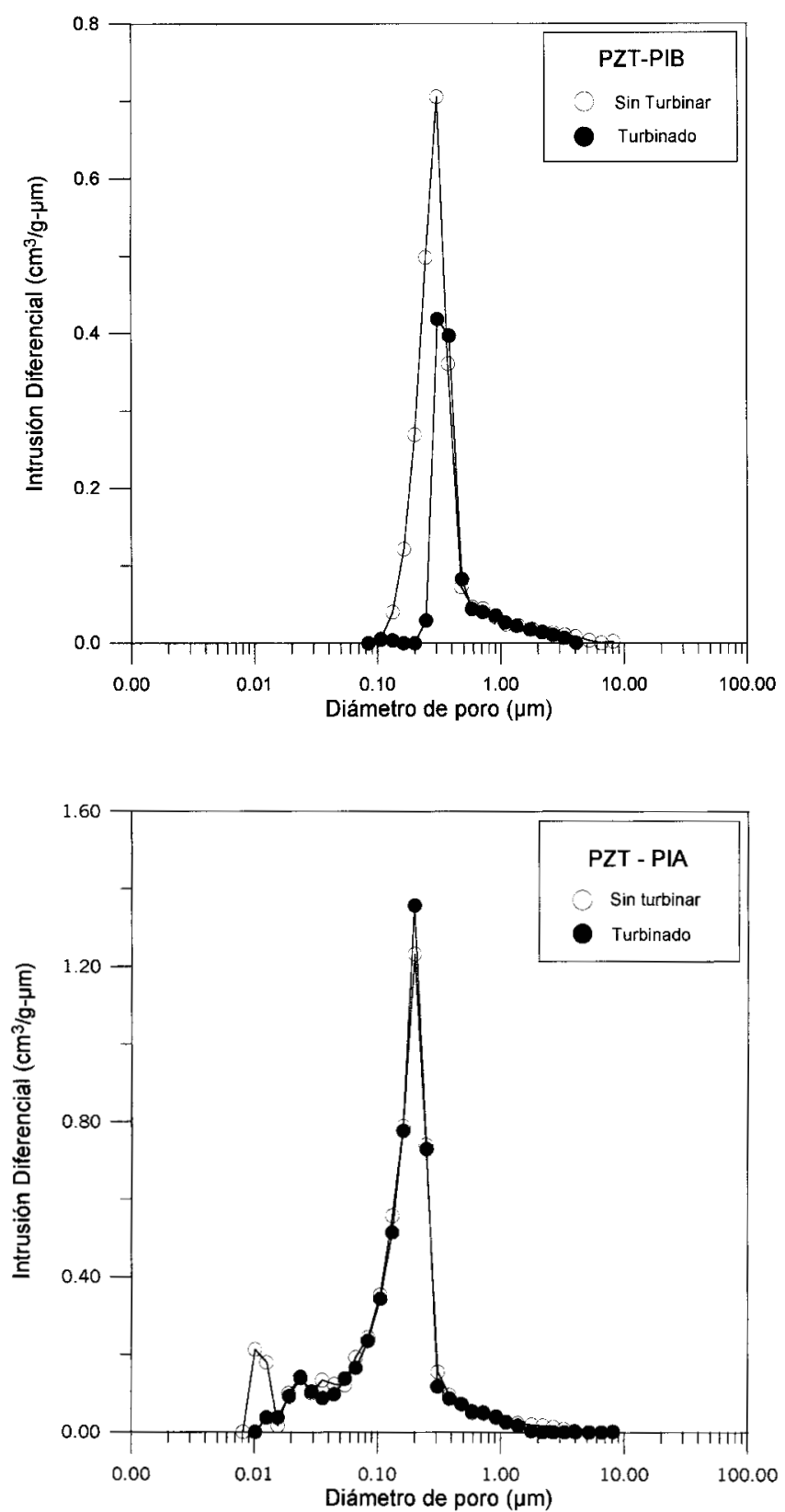

Figura 2. Distribuciones de tamaño de poro antes y después de turbinarlo, correspondientes al polvo cerámico a) PZT-PIB, b) PZT-PIA.
Cuando los polvos cerámicos se someten a un proceso de turbinado a alta velocidad, los macroporos no experimentan modificación, debido a que corresponden a aglomerados blandos que se formaron durante el proceso de secado y tamizado. El proceso de turbinado produce una buena dispersión de las partículas debido al efecto de cizalla, dando como resultado un mejor empaquetamiento del polvo. La cantidad de mesoporos de menor tamaño, en el polvo cerámico PZT-PIB (Figura 2a), se reduce. En las partículas de PZT de mayor volumen de porosidad (Figura 2b) solo se experimenta una ligera reducción del volumen de microporosidad, lo que indica una mayor cohesión de las partículas.

Las características de estos polvos recogidas en la Tabla I indican que poseen un tamaño de partícula similar de $0.3 \mu \mathrm{m}$, siendo estos valores inferiores a aquellos obtenidos por vía convencional de mezcla mecánica de óxidos (17-18). La distribución de tamaños de partícula es bimodal (Figura 3). Los tamaños de partícula superiores a $1 \mu \mathrm{m}$ se corresponden con aglomerados. La mayor homogeneidad del polvo PZT-PIM se atribuye al procesamiento seguido, donde una molienda intermedia favoreció la efectividad del proceso de molienda final.

La distribución más homogénea de poros del polvo PZT-PIB junto con el estado menos cohesionado de sus aglomerados señalan a este tipo de polvo como el más indicado para abordar procesos de modificación superficial (19), debido a que la superficie de sus partículas permite una distribución más uniforme del dopante, a fin de lograr un mejor control de los procesos de sinterización en PZT (20).

Independientemente del tipo de distribución de porosidad, los diferentes polvos cerámicos alcanzaron su máxima densificación para un tratamiento térmico de sinterización de $1150^{\circ} \mathrm{C} / 2$ horas. La densificación se mantiene elevada en un intervalo de temperaturas de $1050^{\circ} \mathrm{C}$ a $1150^{\circ} \mathrm{C}$ (Figura 4). A partir de $1150^{\circ} \mathrm{C}$ el grado de densificación se reduce considerablemente. Los polvos cerámicos obtenidos mediante un solo proceso de síntesis, PZT-PIB y PZT-PIA, presentan una ten-

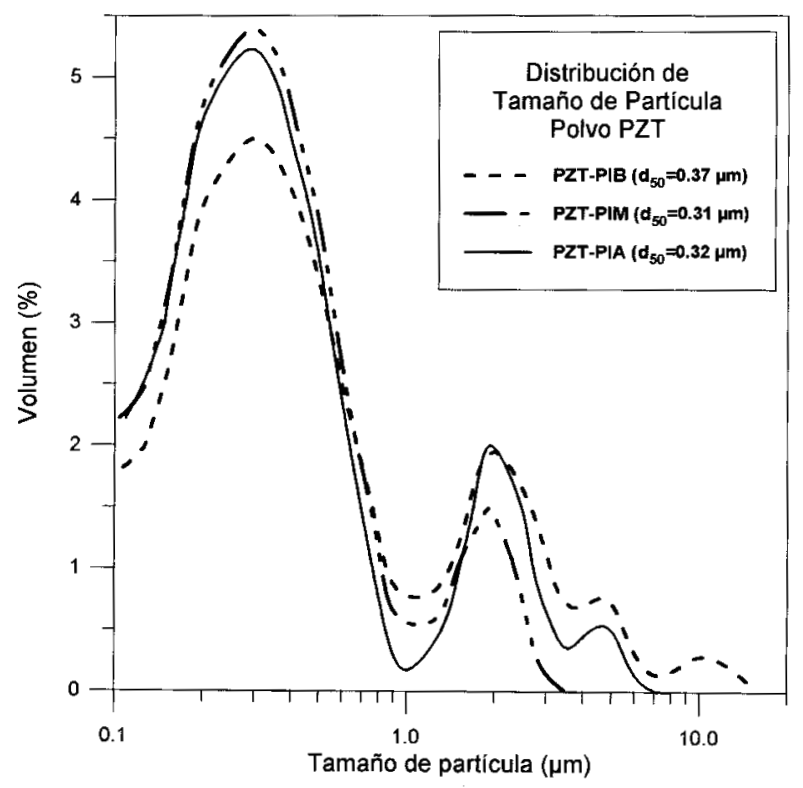

Figura 3. Distribuciones de tamaño de partícula correspondientes a los tres polvos cerámicos PZT obtenidos. El tamaño de partícula para el 50\% de la distribución, d50, se indica en la figura para los tres tipos de polvo. 
dencia de la curva de densificación similar hasta la temperatura de máxima densificación. Sin embargo, a partir de dicha temperatura, $1150^{\circ} \mathrm{C}$, la disminución de la densificación es más acusada en PZT-PIA. Estos procesos están asociados con las pérdidas de peso que tienen lugar durante la sinterización (Figura 5), de forma que una mayor pérdida de peso intensifica el proceso de disminución de densidad del material, ocasionando un aumento de la pendiente de la rama derecha de la curva de densidad, observada a partir de la temperatura de máxima densificación. Se observa a la temperatura de $1250^{\circ} \mathrm{C}$ una elevada pérdida de peso debida principalmente a volatilización de $\mathrm{PbO}$, de $\sim 5.9 \%$ en el material PZT-PIA, $~ 4.1 \%$ en el PZT-PIM y 2.6\% en el PZT-PIB. El polvo cerámico PZTPIM alcanza una mayor densificación en un intervalo amplio de temperaturas entre $1050^{\circ} \mathrm{C}$ y $1150^{\circ} \mathrm{C}$ (Figura 4), puesto que posee una distribución de tamaño de partícula más homogénea que favorece la densificación del material. Ajustando a una recta los valores de densificación del material a partir de $1150^{\circ} \mathrm{C}$, el valor de la pendiente es mayor en el material PZTPIA $\left(5.6 \times 10^{-3}\right)$ y menor en el material PZT-PIB $\left(9.54 \times 10^{-4}\right)$, en correspondencia con la variación de pérdida de peso de los tres materiales, de acuerdo a la siguiente ecuación lineal:

$$
\Delta \mathrm{w}=679(\Delta \rho / \Delta \mathrm{T})+0.85
$$

donde $\Delta \mathrm{w}$ es la variación de peso y $(\Delta \rho / \Delta \mathrm{T})$ es la variación de densidad con la temperatura (Figura 6).

A su vez, teniendo en cuenta que los procesos de pérdida de peso por volatilización de $\mathrm{PbO}$ guardan una estrecha relación con la cantidad de superficie en contacto con la atmósfera y, por tanto, con el área de poros interconectados, es de esperar alguna relación entre la pérdida de peso y el área total de poros de estos materiales cerámicos. Efectivamente, si se representan los valores de las variaciones de pérdida de peso (a partir de $1200^{\circ} \mathrm{C}$ ) en función del área total de poros $\left(\mathrm{A}_{\mathrm{p}}\right)$, para los tres tipos de polvo cerámico (Figura 6), encontramos la siguiente ecuación lineal:

$$
\Delta \mathrm{w}=1.56^{*} \mathrm{~A}_{\mathrm{p}}-1.52
$$

que manifiesta el control que ejerce el área total de poros del material cerámico PZT sobre las pérdidas de peso por volatilización del $\mathrm{PbO}$.

Combinando estas dos ecuaciones [1] y [2] se encuentra la relación lineal:

$$
\mathrm{A}_{\mathrm{p}}=434(\Delta \rho / \Delta \mathrm{T})+1,53
$$

que también expresa el control que ejerce el área total de poros sobre los fenómenos de disminución de densidad en estos materiales.

En la Tabla II se recogen los valores de los parámetros microestructurales, dieléctricos y piezoeléctricos de los materiales cerámicos PZT a partir de las tres distribuciones de porosidad. El tamaño de grano promedio aumenta con el volumen de porosidad inicial. Sin embargo, el tamaño de grano del PZTPIM presenta un valor inferior debido a la distribución mas uniforme de tamaño de partícula del polvo inicial. Los materiales PZT-PIB y PZT-PIA, que presentan un nivel equivalente de densificación a $1150^{\circ} \mathrm{C} / 2 \mathrm{~h}$, exhiben valores similares de sus propiedades dieléctricas y piezoeléctricas. Unicamente el valor de constante dieléctrica presenta un valor mayor en el material PZT-PIB. El material PZT-PIM presenta mejores propiedades dieléctricas y piezoeléctricas, como resultado de una mejor

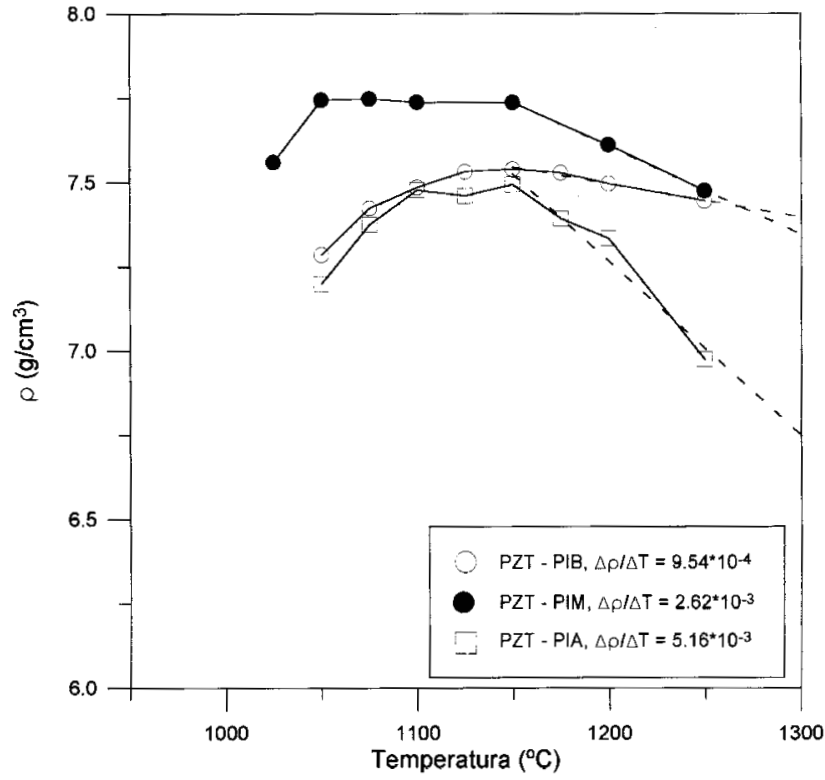

Figura 4. Curvas de densidad en función de la temperatura de los tres materiales cerámicos PZT sinterizados.

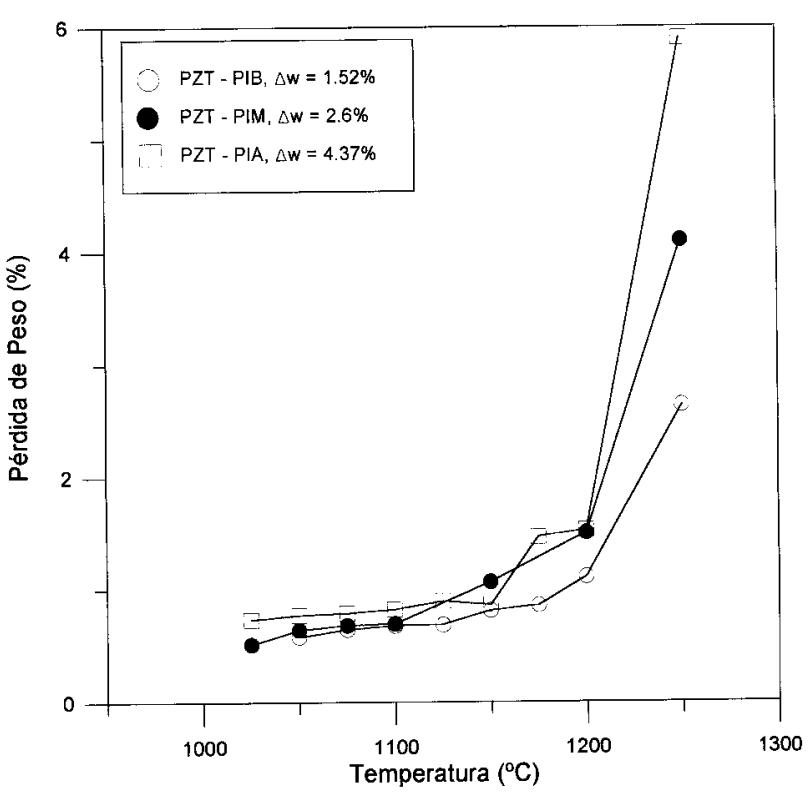

Figura 5. Curvas de pérdida de peso en función de la temperatura de los tres materiales cerámicos PZT sinterizados. 


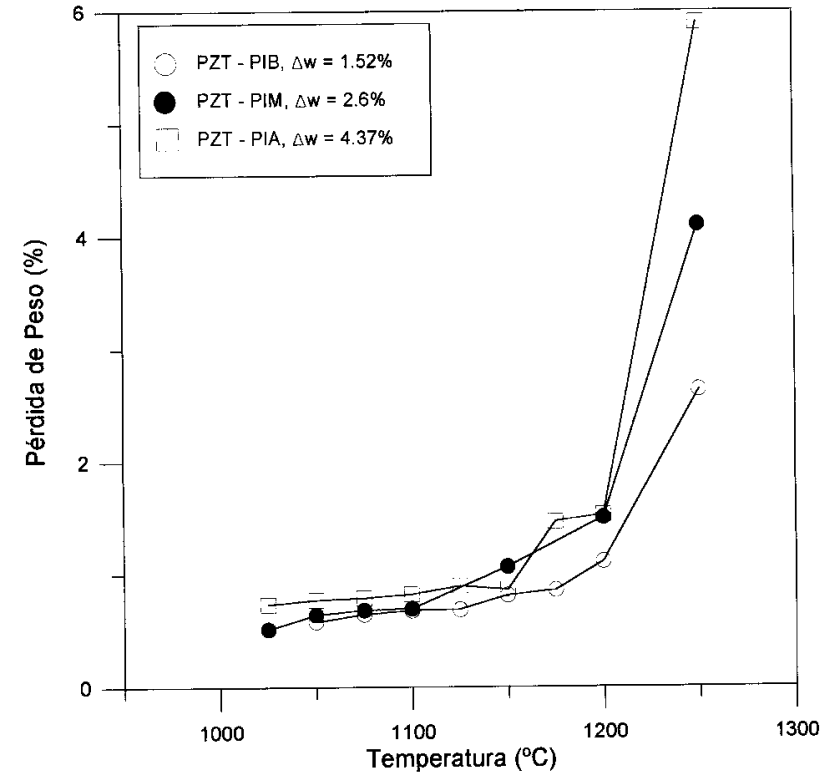

Figura 6. Curvas de variación de la pérdida de peso $(\Delta \mathrm{W})$ y área total de poros del polvo inicial $(\Delta \mathrm{p})$ en función de la variación de la densidad con la temperatura $(\Delta \mathrm{p} / \Delta \mathrm{T})$, para los tres materiales cerámicos PZT.

densificación con respecto a los otros dos materiales (21), siendo éstas sensiblemente superiores a las observadas en materiales tipo PZT 5A, que se presentan como valores de referencia.

\section{CONCLUSIONES}

Empleando el método de calcinación reactiva en dos etapas se han obtenido polvos cerámicos PZT de tamaño de partícula submicrónico de $\sim 0.3 \mu \mathrm{m}$ a partir de óxidos.

Las partículas que constituyen los polvos cerámicos de PZT obtenidos en este trabajo son aglomerados de partículas primarias. La naturaleza de estos aglomerados es diferente según el tipo de porosidad presente en el polvo cerámico PZT. El polvo cerámico que presenta microporosidad está constituído por aglomerados cementados cuyas partículas primarias están fuertemente cohesionadas. Por el contrario, la ausencia de microporosidad en el polvo cerámico indica que éste está constituído por aglomerados blandos de partículas primarias.

Los procesos de densificación del polvo cerámico correspondiente a las tres distribuciones de porosidad obtenidas muestran una estrecha relación con los parámetros de procesamiento. Además se ha observado una correlación lineal entre los procesos de disminución de densidad con la pérdida de peso del material y el área total de poros presentes en el polvo cerámico de partida.

\section{AGRADECIMIENTOS}

Los autores agradecen a CICYT la financiación del presente trabajo dentro del proyecto MAT97-0694-C02-01 y al programa CYTED. D. Luis Alberto Celi agradece al programa de becas ICI de la AECI y a FUNDACYT de Ecuador por la ayuda brindada a través de una beca pre-doctoral, así como a la Escuela Politécnica Nacional, de Quito-Ecuador, por su auspicio.
Tabla II. Propiedades Microestructurales, Dieléctricas Y PieZOELÉCTRICAS DE PZT SINTERIZADO A $1150^{\circ} \mathrm{C} / 2 \mathrm{H}$

\begin{tabular}{|l|c|c|c|c|}
\hline Material & PZT-5A $^{\left({ }^{(}\right)}$ & PZT - PIB & PZT - PIM & PZT - PIA \\
\hline Densidad $\left(\mathbf{g} / \mathbf{c m}^{3}\right)$ & 7.7 & 7.5 & 7.7 & 7.5 \\
\hline Tamaño de grano $(\mu \mathrm{m})$ & $\mathrm{xxx}$ & 4.0 & 3.5 & 5.1 \\
\hline $\mathbf{K}_{\mathbf{p}}$ & 0.60 & 0.60 & 0.67 & 0.58 \\
\hline $\mathbf{d}_{33}(\mathbf{p C} / \mathrm{N})$ & 374 & 380 & 521 & 383 \\
\hline Cte. Dieléctrica $\mathbf{K}_{\mathbf{3}}{ }^{\top}$ & 1700 & 1530 & 2028 & 1444 \\
\hline Qm & 75 & 91 & 75 & 86 \\
\hline Pérdida Dieléctrica (\%) & 2 & 1.5 & 1.7 & 1.4 \\
\hline
\end{tabular}

* Morgan Matroc, Inc.

\section{BIBLIOGRAFÍA}

1. B. Jaffe,W. R. Cook Jr., y H. Jaffe, "Piezoelectric Ceramics". Academic Press, London, U.K. y New York (1971).

2. K. Uchino, "Electrostrictive Actuators: Materials and Applications". Am. Ceram. Soc. Bull., 65 [4] 647 - 52 (1986).

3. L. M. Levinson, "Electronic Ceramics Properties, Devices, Applications". pp. 371 - 492. Marcel Dekker, New York (1987).

4. J. F. Fernández, P. Durán, C. Moure, “Materiales cerámicos ferroeléctricos y sus aplicaciones". Bol. Soc. Esp. Ceram. Vidrio, 32 [1], 5-15 (1993).

5. G. H. Haertling, "Ferroelectric Ceramics: History and Technology". J. Am. Ceram. Soc. 82 [4] 797-818 (1999).

6. J. F. Fernández, C. Moure, M. Villegas, P. Durán, M. Kosec, G. Drazic, "Compositional Fluctuations and Properties of Fine-Grained AcceptorDoped PZT Ceramics". J. Eur. Ceram. Soc., 5 (1998).

7. S. K. Saha y D. C. Agrawal, "Compositional Fluctuations and their influence on the properties of lead zirconate titanate ceramics". Am. Ceram. Soc. Bull., 71 (9), 1424-1428 (1992).

8. I. K. Lloyd, M. Kahn y S. Lang, "Effect of microstructural heterogeneities on piezoelectric behaviour of PZT ceramics". En Ceramic Transaction Vol.8, Dielectric Ceramic: Composition, Processing and Properties, ed.H. C. Ling y M.Yan.The American Ceramic Society, Westerville, OH, 390-398 (1990).

9. K. H. Hardtl y H. Rau, "PbO vapour pressure in the $\mathrm{Pb}\left(\mathrm{Ti}_{1-\mathrm{x}} \mathrm{Zr}_{\mathrm{x}}\right) \mathrm{O}_{3}$ system". Solid State Comm., 7, 41-45 (1969).

10. T. Shrout, P. Papet, S. Kim, G. Lee, "Conventionally Prepared Submicrometer Lead-Based Perovskite Powders by Reactive Calcination". J. Am. Ceram. Soc. 73 [7] 1862-67 (1990).

11. S. L. Swartz y T. R. Shrout, "Fabrication of Perovskite Lead Magnesium Niobate". Mat. Res. Bull.,17,1245-1250 (1982).

12. T. Yamamoto, “Optimum Preparation Methods for Piezoelectric Ceramic and their Evaluation". Ceram. Bull., 71 (6) 978-85 (1992).

13. O. Babushkin, T. Lindback, J.-C. Luc y J.-Y. Leblais, "Kinetic Aspects of the Formation of Lead Zirconium Titanate". J. Eur. Ceram. Soc., 16 1293-1298 (1996).

14. IEEE Standards on Piezoelectricity, ANSI/IEEE Std. 176. The institute of Electrical and Electronics Engineers, New York (USA) (1987).

15. S. Tashiro, N. Sasaki, Y. Tsuji, H. Igarashi, K. Okazaki, "Sintering of Submicron Powders Prepared by Ball Milling". Jap.J.Appl.Phys., 26 Supplement 26-2, 142-144 (1987).

16. M. C. Kerr y J. S. Reed, "Comparative Grinding Kinetics and Grinding Energy during Ball Milling and Attrition Milling". Am. Ceram. Soc. Bull., 71 (12), 1809-1816 (1992).

17. J. F. Fernández, C. Moure, "Sinterización a baja temperatura y desarrollo microestructural de materiales cerámicos PZT obtenidos a partir de diferentes precursores". Bol. Soc. Esp. Ceram. Vidr. 27, 17-23 (1988).

18. G. Kleer, H. Schmitt, "The grain sizes of mixed oxide and chemically prepared PLZT-powders". Mat. Res. Bull., 16, 1541-1544 (1981).

19. J. F. Fernández, A. C. Caballero, P. Durán, C. Moure, “Improving Sintering behaviour of $\mathrm{BaTiO}_{3}$ by small doping additions". J. Mater.Sci., 31, 975-981 (1996).

20. L. A. Celi, A. C. Caballero, M. Villegas, P. Durán, C. Moure, J. F. Fernández, "Microestructura y Propiedades de Materiales Cerámicos tipo PZT con Control de Crecimiento de Grano". Bol. Soc. Esp. Cerám. Vidrio, 38 [5] 487491 (1999)

21. W.E.Lee, W.M.Rainforth, “Ceramic Microstructures Property Control by Processing". Edit. Chapman and Hall, $1^{\mathrm{a}}$ ed.,Cambridge,Great Britain, pp. 67$121,1994$. 